\title{
Simulation of Knowledge Transfer Based on Complex Network
}

\author{
Hailian Liu ${ }^{1}$, Shizhong $\mathrm{Ai}^{2}$ \\ ${ }^{1}$ School of Economics and Management, XIDIAN UNIVERSITY, 266 Xinglong Section of Xifeng Road, Xi'an, Shaanxi 710126, China \\ ${ }^{2}$ School of Economics and Management, XIDIAN UNIVERSITY, 66 Xinglong Section of Xifeng Road, Xi'an, Shaanxi 710126, China
}

\begin{abstract}
Knowledge rigidities in firms make them stick to existing knowledge and impede the inflow of new external knowledge. This paper explores knowledge transfer process among agents from a firm's knowledge rigidity perspective, which helps investigate the factors inducing knowledge lock-in. To conduct a simulation of knowledge transfer process in network with scale-free characteristics, state variables, such as network's degree distribution, firm's knowledge rigidity and so on, are defined. Knowledge transfer rule is also designed. The simulation data and analysis results show that the firms' knowledge rigidities influence the knowledge transfer in networks significantly. Controlling firms'knowledge rigidities and building diversified learning and cooperation relationships in different channels can expand the scope of the knowledge transfer and improve its effectiveness.
\end{abstract}

Keywords:absorptive ability; disseminative ability; knowledge rigidity; complex network; learning curve

\section{Introduction}

In the past few years, researchersand managers have a growing interest in knowledge management, so that the research of knowledge flow and transfer is popular in the field of knowledge management, organizational learning and innovation [1-3].On another front of research, the theory of complex networks provides a useful tool for investigating the dynamics of various kinds of networks [4-7], including computer networks [8], social networks [9] and many others. Some works have addressed the development of knowledge using complex networks as a tool [10-11], and concluded that the structure of complex network has high effect on knowledge transfer process.Moreover, the characters of agents, such as absorptive ability, knowledge rigidity and so on, can affect knowledge diffusion process too. Nevertheless, how the relative knowledge absorptive ability and the knowledge rigidity of agents influence the dynamic process, and how to measure the two characters, there are no results so far.

In this paper, we use complex network to present a new model in which agents havecharacters such as knowledge rigidity, absorptive and disseminative ability, and have links with other agents which can send knowledge to them. And then we observe the knowledge transfer process in the complex network by controlling the network's structure and agents' characters.

\section{Research Design}

The model we chose to describe knowledge transfer is scale-free network because of knowledge network's heterogeneity. Based on Xi and Tang [12], the network G can be represented by graphs, a knowledge agent of the network can be denoted by the agent $v_{i}$, and a knowledge transfer channel between agents $i$ and $j$ can be denoted by their link $\mathrm{e}_{i j}$. In the knowledge network, every agent has a certain level of knowledge absorptive and disseminative capability and knowledge rigidity. Among agents, knowledge spreads through their links. And during every period of evolution time, knowledge diffusions take place in the network. Agent i can absorb knowledge from its neighborj through the link between $i$ and $j$ only if they have enough ability. Thus, a knowledge network can be represented as:

$\mathrm{G}=(\mathrm{V}, \mathrm{E})$ represents the topology of the network.

$\mathrm{V}=\left\{\mathrm{v}_{1}, \mathrm{v}_{2}, \ldots, \mathrm{v}_{i}, \ldots, \mathrm{v}_{n}\right\}$ denotes the set of $\mathrm{n}$ agents $E=\left\{e_{i j}, i \neq j\right\}$ is the set of their links

The network parameters mainly include the agents' number n, the degree distribution exponent of scale-free network, agents' interaction rules, and rules that can change knowledge status quo of the agents.

\subsection{The rules of agents' properties}

In this section, the agents' properties which involved in the process ofknowledge transfer evolution are presented.

1) Knowledge state. Any agent can be represented by an intelligent actor who has limited knowledge of specific problems because of his or her bounded cognitive rationality. Thus, we assume that there are two knowledge state of a specific problem or task for each agent, the initial input condition of 0 or 1 . The agents that have absorbed the specific knowledge are denoted by the number " 1 ", and the others are denoted by " 0 ".

2) Absorptive ability.Absorptive ability refers to the organization identifying and recognizing the value of external knowledge and information, absorbing and assimilating knowledge and information, and putting them into application [13]. Zahra [13] thinks absorptive ability is a set of organizational convention and process, and its essence is the skills of managing external knowledge. Therefore, whether knowledge transfer process succeeds depends on largelythe value of anagent's absorptive ability. If the agent's absorptive ability is too low to absorb other knowledge, knowledge transfer can't take place. We use $A_{i}$ to represent that each agent has ability to change its knowledge state [14], and have its initial value provided by $A_{i}=\operatorname{rand}[0,1]$. 


\section{International Journal of Science and Research (IJSR) \\ ISSN (Online): 2319-7064}

Index Copernicus Value (2013): 6.14 | Impact Factor (2015): 6.391

3) Disseminative ability. Disseminative ability means the ability of network members (knowledge holders) to efficiently and effectively codify, articulate, communicate and teach knowledge to other network members [14]. We present the capacity of each agent to express and communicate its current knowledge to other agentby $\mathrm{D}_{i}$. And its initial value is provided by $D_{i}=\operatorname{rand}[0,1]$.

4) Knowledge rigidity. Knowledge rigidityis an invisible force inducing the firm to hold and use specific knowledge for quite a long time, and impeding a firm to adapt to a changing environment [15]. With satisfaction of the benefit from the specific knowledge, the preference for that rigid knowledge becomes stronger. The more frequently the rigid knowledge is used, the more intensifying its rigidity will be.Needless to say, individuals' learning effects increases by S-curve. Therefore, we choose learning curveto represent the knowledge rigidity in our research. It follows the equation

$$
\mathrm{R}_{\mathrm{i}}=\left(1+\alpha^{*} \exp \left(-\beta^{*}\left(\mathrm{t}-\mathrm{t}_{0}\right)\right)\right)^{-1}
$$

Where $\alpha, \beta$ are the parameters of learning curve (Learning curve is a process where people develop a skill by learning from their mistakes. A steep learning curve involves learning very quickly. Under the condition that $\beta$ which is a fixed value, the lessthe $\alpha$, the more steep is learning curve. On the contrary, when $\alpha$ is invariable, with increasing value of $\beta$, the learning efficiency increases.), and it will change with the change of individual, $t_{0}$ is the time when agent absorbs new knowledge from its neighboring agents, $\mathrm{t}$ is current time. $R_{i}$ denotes the knowledge rigidity and it is incremental over time. Normally, one agent cannot be fully depended on onekind of knowledge no matter how it prefers the knowledge. So, $R_{i}$ is typically smaller than 1. Once an agent accepts new technology, its rigidity of the new knowledge will become 0 and the current time will be set to $t_{0}$. For example, we let $\alpha=200, \beta=0.1$, agent 5 upgrades new technique at time 5 , its rigidity of the new technique at time 10 isR $_{5}=(1+200 * \exp (-0.1 *(10-5)))^{-1}($ As shown by Figure 1$)$.

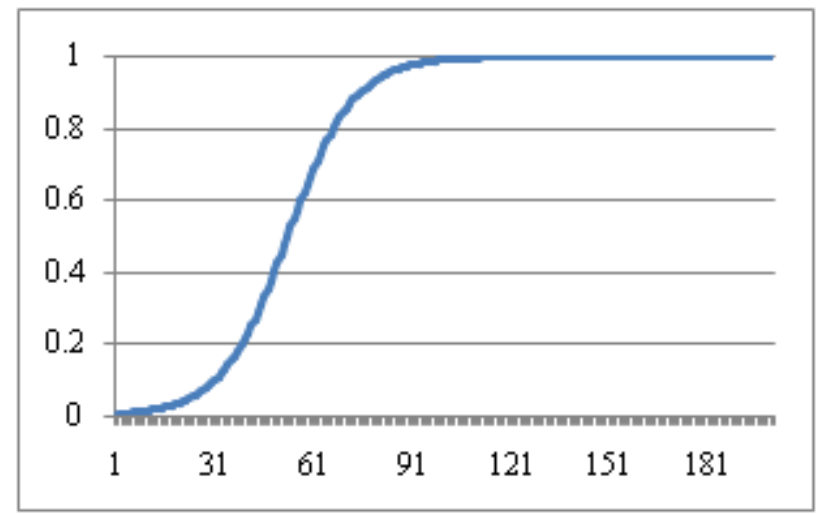

Figure 1:Learning Curve with $\alpha=200, \beta=0.1$

\subsection{The interaction rules of agents}

This section presents the interaction rules of agents in the knowledge network and describes how agents interact with each other.

1) The knowledge state of agent affects the knowledge state of its directly linked neighbor agents. By the same token, the knowledge state of each agent is affected by the knowledge state of its directly connected neighbors.

2) The impact rule of knowledge rigidity.

The influence of knowledge rigidity is different because of different role the firm plays in knowledge transfer process. The technology acceptance model proposed by Davis, thinks a technology was considered useful and easy to use will be accepted and put into use by the members of organization. Firm agents in knowledge network cannot judge the value of new knowledge until they have a thorough practice, and there will be knowledge rigidity in this process. Once they are considered to be valuable and easy to practice, that is, to have knowledge rigidity, firms will transfer them to other agents in the network.

To acquire new knowledge is one of the major methods that enterprises keep the industry status and gain competitive advantage. However, whether to accept the new knowledge is affected by enterprises' knowledge rigidity. If the firm has a great dependence on the existing technology, namely the value of knowledge rigidity is high; it will not choose to absorb the new knowledge. So, suppose the agents' interaction rule affected by rigidity as follows:

$v_{i}$ recieve decision $=\left\{\begin{array}{l}1, \text { if } R_{i}<R r_{\text {max }}, \\ 0, \text { if } R_{i} \geq R r_{\text {max }}\end{array}\right.$

$v_{j}$ send decision $=\left\{\begin{array}{l}1, \text { if } R_{j}>R s_{\text {min }} \\ 0, \text { if } R_{j} \leq R s_{\text {min }}\end{array}\right.$

Where $v_{i}$ denotes knowledge receiver, $v_{j}$ is sender, $R_{i}$ and $R_{j}$ denotes the knowledge rigidity of receiver and sender respectively, $R r_{\max }$ is the maximal knowledge rigidity that enterprises are willing to absorb new knowledge, in other words, when enterprises' rigidity is greater than $R r_{\text {max }}$, they won't accept new knowledge. Similarly, $R s_{\min }$ denotes the minimum knowledge rigidity that enterprises' must reach, when they can't achieve the point, they won't send their knowledge to others. We use symbol " 1 " to represent receive and send knowledge, and " 0 " means refuse to do that.

3) The effect of knowledge absorptive and disseminative ability

The change of current knowledge of each agent is determined by the initial knowledge state of its directly connected agents, the relative absorptive capacity and disseminative capacity between its neighbors and itself, and its knowledge rigidity. Supposing the disseminative capacity of agent $\mathrm{i}$ is $\mathrm{D}_{i}$, the absorptive capacity is $\mathrm{A}_{i}$, the agent's current knowledge state is $S_{i}$. Moreover, suppose directly connected neighbor nodes $i$ and $\mathrm{j}$ interact and communicate with each other, then,

$$
\text { if } S_{j}=1, D_{j}>D_{\mathrm{T}} \text { and } A_{i} \geq A_{T} \text {, then } S_{i}=S_{j}
$$

That is, if $\mathrm{A}_{i}>\mathrm{A}_{T}, \mathrm{D}_{j}>D_{T}$, then $v_{i}$ will absorb the knowledge of $v_{j}$ and its knowledge state will change.

\section{Numerical Simulation Analysis}

\subsection{Settings}

We choose scale-free network as the carrier of knowledge flow network, and do analysis on basis of it.

Randomly generate a scale-free network with the degree 


\section{International Journal of Science and Research (IJSR) \\ ISSN (Online): 2319-7064}

Index Copernicus Value (2013): 6.14 | Impact Factor (2015): 6.391

distribution exponent- 6 and the agents' number-1000. We suppose that knowledge transfer begins randomly from five of 1000 agents.

We randomly distribute values of the absorptive capacity, and disseminative capacity to specific agents under given neighbor agents. $A_{T}$ is set to be 0.3 which means that only $70 \%$ of all the agents can absorb knowledge from neighbors successfully. $D_{T}$ is set to be 0.2 . $R s_{\min }$ and $R r_{\max }$ are set to be 0.2 and 0.8 respectively. We also fix the parameter $\alpha$ of learning curve to 200 and the coefficient $\beta$ to 0.1 .

Agents having new knowledge will transfer it to others according to the given rules. The agents that change their knowledge state in the process are recorded and they will continue to transfer knowledge to its neighbors. In the end, the whole agents will have accessed to this knowledge, but only a part of 1000 agents accept and use it. Thus, the percentage of learned agents within the total number is the knowledge learning rate in this network.

In order to increase the measuring accuracy, the sample averaging method must be introduced, so that a certain time of measurement is needed. Under the same initial condition, we repeat the simulation 100 times and then take the average to analyze the result.

\section{Results}

We propose four kinds of policies to observe the process of knowledge diffusion in scale-free network. We set time step of the simulation to be 100 in Netlogo.

\subsection{Different degree distribution exponent}

In this section, we simulated what would happen if the degreedistribution exponent of the knowledge network were 3, 6 and 9, respectively. We ran the simulation 100 times.

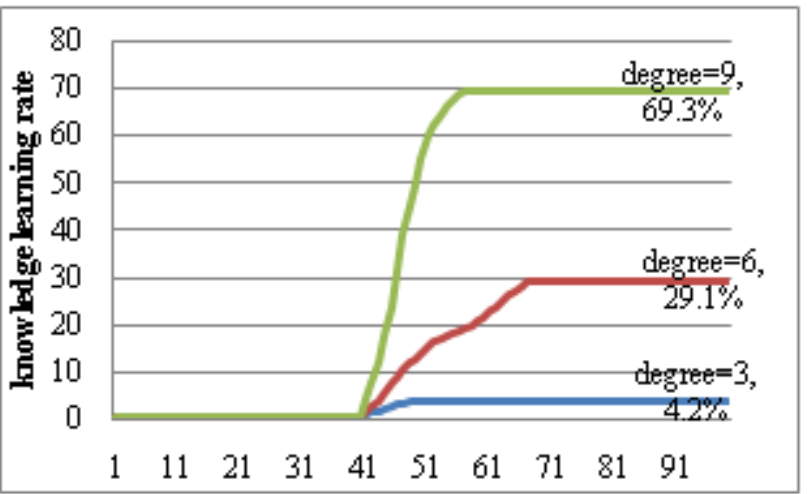

Figure 2: The equilibrium scale after 100 simulations with different degree distribution exponent.

Figure 2 indicates the relationships of network knowledge equilibrium under different parameters. We can see that there is similar trend but slightly variation under those three situations (as shown by Figure 2). The knowledge learning rate stabilizes at $4.2 \%, 29.1 \%$ and $69.3 \%$ for the degree distribution exponent of the network being 3,6 and 9, respectively, over time. Because the degree distribution exponent of network changes, the topology of the network changes, and then the knowledge learning rates of three different networks are variant. The simulation results demonstrate that the bigger the degree of network, the faster will the network knowledge be transferred, and the more the agents accept the knowledge in network. Therefore, we can increase network's knowledge level by intensifying the links between agents.

\subsection{Change the parameter $\beta$ of learning curve}

In the section above, which knowledge transfer processes are carried out under a same learning curve, means that agents have same learning efficiency in that three situations. But what will happen if we change their efficiency in studying. Therefore, we simulate how knowledge equilibrium of a network will change over time by supposing that $\beta$ is $0.05,0.1$, and 0.3 respectively, the degree distribution is 6 .

In the Figure 3, one can see that, the highest learning rate occurs when $\beta$ is 0.05 , namely $\beta$ is minimum. Because the less the $\beta$, the greater the learning efficiency. And great learning efficiency leads great learning rate. Thus, we can conclude that there is a higher learning rate with a lower value $\beta$ of in equilibrium. We can improve the learning achievements by appealing to agents in the knowledge network to raise their own learning capacities.

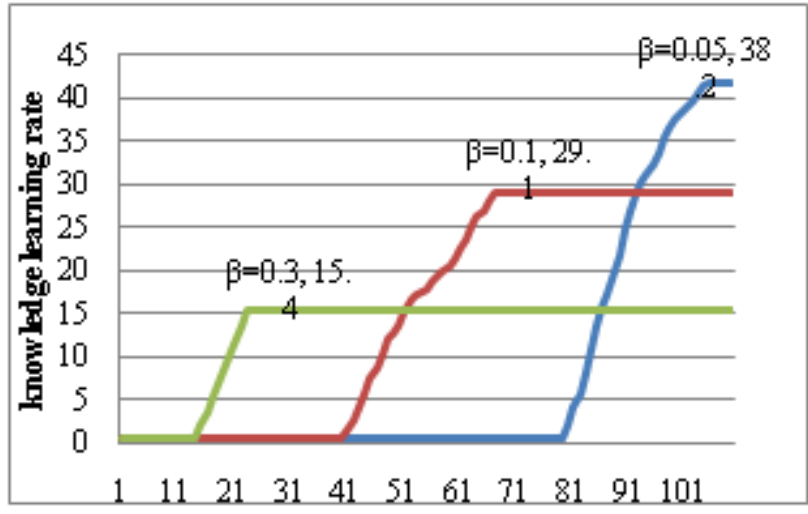

Figure 3: The difference of knowledge equilibrium with variant $\beta$, where degree $=6$

\section{Conclusions}

Our study offers important policy implications to researchers and managers. This paper models knowledge transfer process in a scale-free network, and reveals that both the agents' characters and network's structure affect knowledge transfer process. The speed and achievement of knowledge transfer in network swing so much with the change of the degree distribution. The larger the degree distribution of network, the faster the speed to reach equilibrium, and then the more the number of agents having new knowledge. Therefore, we can improve the knowledge level of network we are in through increasing the links with others especially links between ordinary agents and the hub agents. By the same token, we can raise the connection between other department and administration authority in firms in order to manage efficiently and learn fast.

Besides, the agents' own characters, learning efficiency, has positive impact on the effect of knowledge transfer. Which means that the higher the learning efficiency, the more the 


\section{International Journal of Science and Research (IJSR) \\ ISSN (Online): 2319-7064}

Index Copernicus Value (2013): 6.14 | Impact Factor (2015): 6.391

number of learned agents. It is suitable for the actual situation. If one's learning efficiency is improved, its speed of learn something becomes high. So, firm should try its best to improve its own learning efficiency to absorb new technology faster than before, then gains and holds competitive advantage.

There are many issues arising from on this model that are worth further investigation. For example, this paper has analyzed the absorptive ability, but the disseminative ability and its impact have not been discussed. For the process of absorbing knowledge from neighbors, only the case of random absorption capability is considered. But different absorption capabilities for different agents with different links are more realistic, which will be further studied in our future research.

\section{References}

[1] M. M. Parent, D. MacDonald and G. Goulet"The theory and practice of knowledge management and transfer: The case of the Olympic Games" Sport [J]. Management Review, 2013.

[2] P. H. Hendriks and C. A. Sousa"Rethinking the liaisons between Intellectual Capital Management and Knowledge Management" [J]. Journal of Information Science, 2013, 39(2): 270-285.

[3] Qiang Li. The combination of Human Resource Management and Knowledge Management in E-commerce environment[J]. Management Science and Electronic Commerce. 2011, 4(8): 6432-6437

[4] Choudhary, A,Kohar, V, Sinha, S. Noise enhanced activity in a complex network [J].European Physical Journal, 2014,87(9): 202-210.

[5] Qzkanlar, A, Clark, A.E.ChemNetworks: a complex network analysis tool for chemical systems [J].Journal of Computational Chemistry, 2014, 35 (6): 495-505.

[6] S. Ferretti. Publish-subscribe systems via gossip: a study based on complex networks [J]. Proceedings of the Fourth Annual Workshop on Simplifying Complex Networks for Practitioners, 2012, 12, 7-12

[7] Kashirin, V.V, Ivanov, S.V. Heuristic algorithms for modelling and optimisation of complex network structures [J].Dynamics of Complicated Systems, 2013, 7(3): 41-46.

[8] M.E.J. Newman, Ranking in collaboration networks using a group based metric [J]. Journal of the Brazilian Computer Society, 2011,17 (4): 255-266.

[9] Guopeng Li, ZhisongPan,etc. Community discovery and importance analysis in social network [J]. Intelligent Data Analysis, 2014,18(3), 495-510.

[10] I. Licata, A dynamical model for information retrieval and emergence of Scale-Free clusters in a long term memory network $[\mathrm{J}]$. Emergence: Complexity and Organization, 2009,11 (1): 48-57.

[11] S. Ferretti. Publish-subscribe systems via gossip: a study based on complex networks [J]. Proceedings of the Fourth Annual Workshop on Simplifying Complex Networks for Practitioners, 2012, 12, 7-12.

[12] M, C.W. and L.D. A, Absorptive capacity: A new perspective on learning and innovation [J]. Administrative Science Quarterly, 1990.

[13] George, Z., Absorptive Capacity: A Review
Reconceptualization and Extension [J]. Academy of Management Review, 2002, 17(2):185-200.

[14] Mu, J., F. Tang, and D.L. MacLachlan, Absorptive and disseminative capacity: Knowledge transfer in intra-organization networks $[\mathrm{J}]$. Expert Systems with Applications, 2010, 37(1): 31-38.

[15] Zhou, Z. and Z. Chen, Formation Mechanism of Knowledge Rigidity in Firms [J]. Journal of Knowledge Management. 2011,15(5): 820-835.

\section{Author Profile}

Hailian Liu received the M.S. degree in Managementfrom the School of Economics and Management,XIDIAN UNIVERSITY, China during2010 and 2014. She is now pursuing Masters of

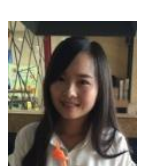
Management Science and Engineering from XIDIAN UNIVERSITY since 2014 under theguidance of Prof. Shizhong Ai. Her specialization area is knowledgemanagement. 